\title{
An Authentication of Significant security for accessing Password through Network System
}

\author{
Syed Umar \\ MLR Institute of Technology \\ Hyderabad \\ India \\ umar332@gmail.com
}

\begin{abstract}
Significant security password to authenticate users on a network system network system for small and large. Text passwords is the standard form of authentication of users on the site for comfort and convenience. In fact, it probably caught on the user's password with various threats and vulnerabilities and threats. ordinary users use text passwords for authentication when they register with the selected account on the site. weak password selected by the user and the application between the sites that a domino effect. Additionally, enter the password of the computer thief a password is not a reliable threat arrival could begin passwords, such as phishing, key loggers and capturing malware attacks steal. OPASS introduce more help with a specific user authentication protocol in this paper. The concept of the universal system and methods of organizations and users to implement password policies. The system is designed for user authentication protocols OTP relating to benefit the users of mobile phones and short message services giant passwords and reuse of passwords to steal a series of attacks. OPass a live phone number of each contribution, this unique recreation and telecom service providers involved have shot three OPASS, users need to log in a password on any computer in the long-term prototype website. After remembering OPASS, we believe OPASS effective and inexpensive compared to a conventional web authentication mechanism.
\end{abstract}

Keywords: Security, Authentication, Messages, OPass, Networking, Password

Received: 5 September 2018, Revised 12 November 2018, Accepted 28 November 2018

DOI: $10.6025 / \mathrm{jisr} / 2019 / 10 / 1 / 18-23$

(C) 2019 DLINE. All Rights Reserved

\section{Introduction}

If the public network, since most of the activities available on the Internet, the user authentication is the most important part of the security in the area. text password is used as the primary user authentication method for the last twenty years. To register, select the site a user name and password. So as you can after you log on to a successful web page, the user must confront noted passwords. In general user authentication based on a password dictionary attacks and violence when users select passwords. However, users who have difficulty learning the encrypted text to have by heart. Users select a password, they can easily remember that they too can know the password unsafe. Crucial problem is that they can view the same password on different 
pages. [1] Can lead reuse passwords users lose their sensitive data is stored in different places, if a hacker compromised a good password. This attack code usually strikes back. The problems that caused the negative effects on the human factor. When designing the user authentication, account vitals' human factor. Alternatively, use password graphs [2], [3], [4], and other password management devices [5], [6] as well as three authentications. However, the password cannot solve the practical graphics. important for flying as phishing attacks to watch [4] In addition to reuse the attacks. While there is much research in the online protection of passwords used performed [5] [2] and other websites dictionary attacks [8] many visualizations hash used [7] The defence is still limited in accuracy and efficiency.

In this article, we want a password and a second attack with stolen user authentication protocol called OPASS password [1] the use of the mobile phone users will avoid using reuse generate unique passwords and short message service used to send the message. OPASS main concept is the user having to remember to authenticate quite a password every traditional PC or types. A basic user authentication is made with new components OPASS unique passwords and telephone communication channel used to send messages Active Authentication, SMS is now used.oPass benefits.

- Protection against phishing Sometimes forged to connect with their deceptive phishing attacks users of the site. Users are invited to OPASS resist phishing attacks.

- Anti-malware sensitive information to take the user's password (e.g. keyloggers). In OPASS, users can go to another place, malware without a password on their computers may not mentioned here or malware.

- Recording and safe recovery OPASS, communications interface together SMS. OPass obligations of telecommunications service provider (TSP) to accordingly find the correct number of users and websites. OPASS SMS help establish a secure channel for the exchange of messages in programming and the recovery period. The recovery handle the event to design a user loses their phone. With the new support of the SIM card, OPASS work on new phones.

- Password reuse prevention and weak password Avoidance- OPASS approach unique password. For each phone connection automatically illuminate the unique passwords that do not need to remember the password.

\section{Details of the Implementation}

This process is a movement of the user authentication to prevent the reuse of phishing and password. The purpose of the protocol is to prevent the user to write their passwords on a public kiosk. By adopting the unique password, the password information is no longer needed. One-time password transmitted when the user terminates an existing session. various Internet channels via SMS mobile phone users to prevent password theft. We believe that SMS is safe and appropriate to request the relevant information between mobile phones and websites. Based on SMS, the user is authenticated by the site without entering a password and do not block the trust. user password is used to restrict access to the phone user. In this process, each user to store a password cell phone just for long-term access. The former use of the passes of mobile phone theft security information password. The idea that the system is as follows.

[1] Each Web server has a single telephone number. From the SMS channel, the user can interact with the website using a phone.

[2] Telecommunications services to participate in the recording and recovery. Module TSP is the link between the client and the Web server on the server. It provides services to customers for the recording and recovery of each Web service, for example, customer feedback and the incorporation phase identifier ID to kill the web server. Finally, the module sends TSP numbers and customer demand and the receiving server.

[3] The client (e.g., users) a compound of the teaspoon server module over a $3 \mathrm{G}$ connection.

[4] If a user loses his phone, he can stop his service provider (c) to disable the SIM card is wrong and create a new card with the phone. Therefore, the user ends the recovery phase. 3. System.

\section{The Module Analysis and Usage in}

There are three modules:

- Registration phase.

- Sign warns.

Journal of Information Security Research Volume 10 Number 1 March 2019 


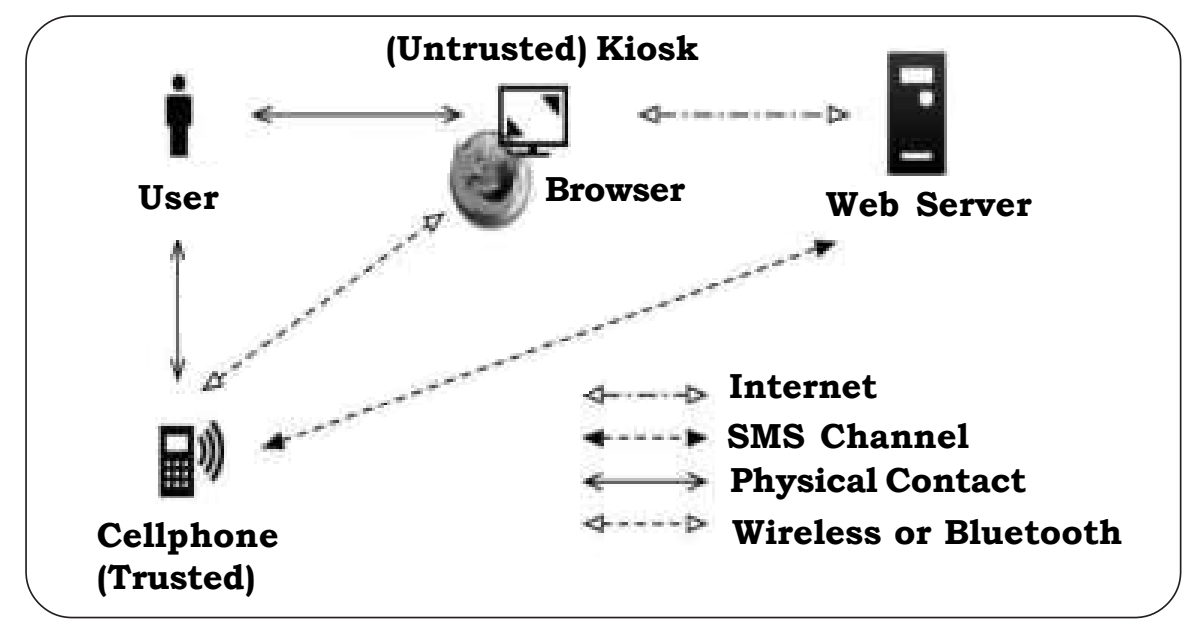

Figure 1. Total architecture of proposed system

- Recovery Phase

3.1 Phase of Registration

- User enter the user ID and the server ID.

- You Cell-phone send a TSP

- Sends the user ID, the phone does not work and a shared server.

- Server through the information and send it to a TSP.

- TSP sent to the server and share your phone.

- Users enter the old password.

- Cell Phone calculating the secret key to save the record of generating information is sent to the server to check the accuracy.

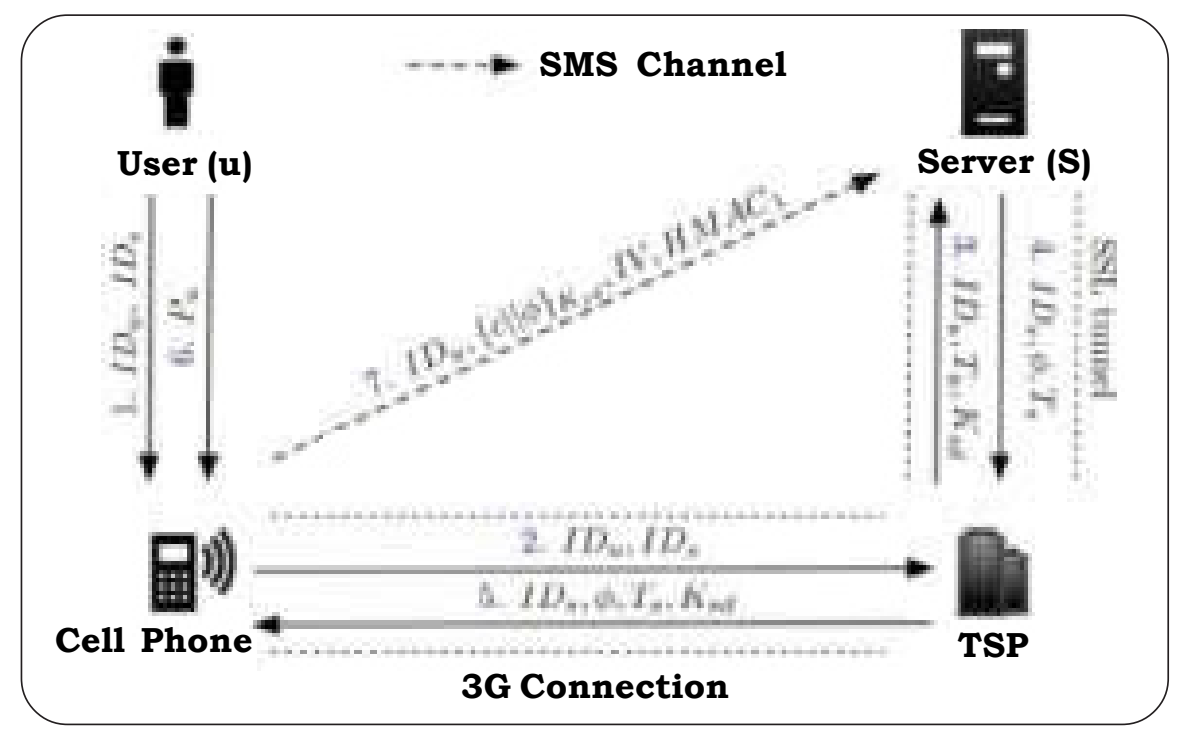

Figure 2. Procedure the registration phase

\subsection{Phase Connection}

- Browser send service requests. 
- Server to verify the information in the database.

- Generate a new nonce.

- Then the phone.

- User going to enter the old password.

- A hobby password to generate a cell phone to connect incoming SMS currently generates nonce.

- Server and verify the authenticity of incoming SMS.

- The server sends the Internet information.

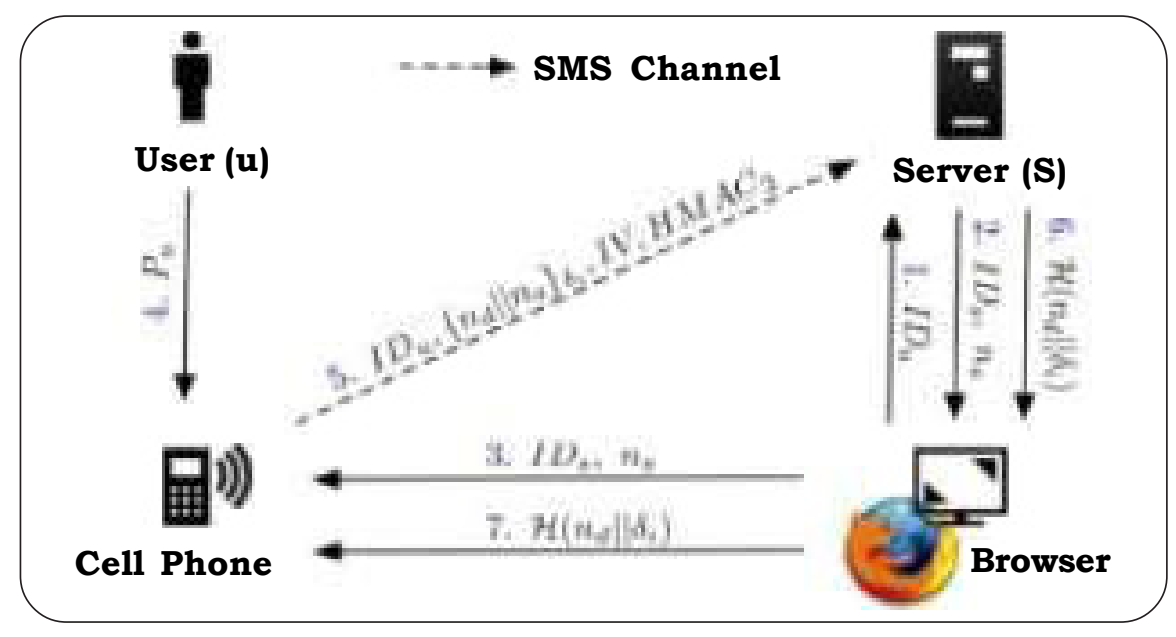

Figure 3. Procedure compound phase

\subsection{Recovery Step}

- User enter the user ID and the server ID.

- You Cell-phone send a TSP

- Sends the user ID, the phone does not work and a shared server.

- The server verifies the existence and the new nuncio and answers c.

- TSP send the same message server.

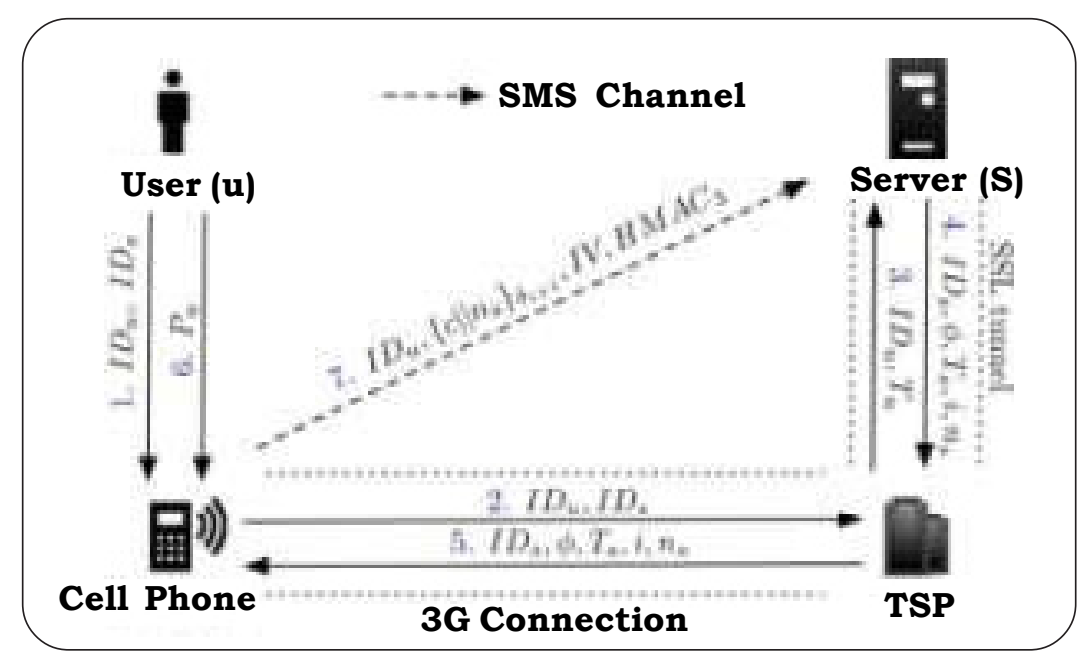

Figure 4. Procedure recovery phase 
- Users enter the old password.

- The phone calculates the secret key to a unique and ready for password restoration workshop sent to the server to generate check the accuracy.

\subsection{Platform}

Windows (Windows 7, Windows XP), programming tools for Android 2.2 SDK and emulator will be installed, empty Eclipse (version 3.5.1 and higher), the SQLite database, Apache, MySQL database. Hardware: Intel Core 2 Duo processor, 1GB of RAM, Android osv2.0 above, a GSM modem. Technology: Java, HTML, XML, Android API, PHP, SMS Lib (open source library).

\section{Conclusion and Future Scope}

Recommended user authentication protocol used cell phones and messaging systems to the principle attacks. The theft and password normal structure of the system to prevent re-use to try to eliminate the negative effects on the human factor. Assume that each number from a single position. Set Telecommunications Service in the recording and participate recovery phases. Through protocol, each user only must protect a long password remind phones. Users can set a password for the untrusted computer to enter to connect each site. Compared to previous systems, this method is the first user authentication protocol passwords at the same time the risk of a decline in attacks and re-steal passwords. Arguing that OPASS take the proposal to the passwords of independence between each protocol strategy. Some online banking situation, the bank provides the user prints on paper a list of OTPs. The user must enter the OTP List for each transaction. In Brazil and in other countries such as Austria, are OTPs are usually marked with $\tan$ (replace "method of authentication transactions). Some banks eventransmit Yet as mobile phone users via SMS, they are called in this case mTAN (for "mobile TAN"). Google has recently launched Mobile OTP line and the ground has received all google accounts.OTP via SMS. Standard could also all mobile users access registered users to use a number (up to 10), the security code previously generated once dynamically generated as a secondary factor in the license OTP, once signed their password.

A phone keeps low cost thanks to its large customer base already different from the OTP generation mobile phone was for the purpose. Space processing power and memory to normal OTPs are not relevant to the elegant and modern camera phones typically require use. Mobile supports multiple signals in a single installation of the program, allowing users the ability to authenticate multiple sources from a single device. These results also provide model- application especially for mobile users. Consequently, our authentication protocol is acceptable and reliable user for the user and safer than the original protocol system.

\section{References}

[1] Hung-Min Sun, Yao-Hsin Chen., Yue-Hsun Lin. (2012). oPass: A User Authentication Protocol Resistant to Password Stealing and Password Reuse Attacks, IEEE Transactions On Information Forensics And Security, 7(2) (April).

[2] Florencio, D., Herley, C. (2007). A large- scale study of web password habits, In: WWW '07: Proc. 16th Int. Conf. World Wide Web., New York, 2007, p. 657-666, ACM.

[3] Chiasson, S., Forget, A., Stobert, E., P. C. (2009). Multiple password interference in text passwords and click-based graphical passwords, In: CCS '09: Proc. 16th ACM Conf. Computer Communications Security, New York, 2009, p. 500-511, ACM.

[4] Wiedenbeck, S., Waters, J., Sobrado, L., Birget, J.-C. (2006). Design and evaluation of a shoulder-surfing resistant graphical password scheme, In: AVI '06: Proc. Working Conf. Advanced Visual Interfaces, New York, 2006, p. 177-184, ACM.

[5] Gawand, S., Felten, E. W. (2006). Password management strategies for online accounts, In: SOUPS '06: Proc. 2nd Symp. Usable Privacy. Security, New York, 2006, p. 44-55, ACM.

[6] Ives, B., Walsh, K. R., Schneider, H. (2004). The domino effect of password reuse, Commun. ACM, 47 (4) 75-78.

[7] Wiedenbeck, S., Waters, J., Birget, J.-C., Brodskiy, A., Memon, N. (2005). Passpoints: Design and longitudinal evaluation of a graphical password system, International Journal of Human-Computer Studies, 63 (1-2)102- 127.

[8] Pinkas, B., Sander, T. (2002). Securing passwords against dictionary attacks, In: CCS '02: Proc. 9th ACM Conf. Computer Communications Security, New York, 2002, p. 161-170, ACM.

[9] Thorpe, J., Van Oorschot, P. (2004). Towards secure design choices for implementing graphical passwords, presented at the 
20th. Annu. Computer Security Applications Conference., 2004.

[10] Jermyn, A., Mayer, F., Monrose, M. K., Reiter, Rubin, A. D. The design and analysis of graphical passwords, In: SSYM'99: Proc. 8thConf. USENIX Security Symp., Berkeley, CA, 1999, p. 1-1, USENIX Association.

[11] Perrig, A., Song, D. Hash visualization: A new technique to improve real-world security, In: Proc. Int.Workshop Cryptographic Techniques-Commerce, Citeseer, 1999, p. 131-138. 